\title{
Depth adjacency and the Ponzo illusion
}

\author{
WALTER C. GOGEL \\ University of California, Santa Barbara, California $9 \$ 106$
}

The effect of depth displacement of test bars from the induction wedge of the Ponzo illusion was investigated in two experiments. Either two wedges of opposite orientation were presented simultaneously, one at a near and the other at a far distance, or only one wedge was presented at either the near or the far distance. The test bars were stereoscopically either in the plane of the wedge or displaced from the wedge in distance. When the two wedges were presented simultaneously, the direction of the Ponzo illusion was determined by the wedge at the same perceived distance as the test bars. When only one wedge was present, stereoscopic displacement of the bars in front of, but not behind, the wedge decreased the magnitude of the illusion. The results are interpreted in terms of the adjacency principle.

In two studies of induced motion, the perceived (stereoscopic) depth between the induction and test object has been found to be a significant variable in determining the magnitude of induced motion (Gogel \& Koslow, 1971) and its direction (Gogel \& Koslow, 1972). These results are interpreted as supporting the adjacency principle which states that the effectiveness of cues between objects in determining perceived object characteristics, e.g., perceived size or motion, is inversely related to the perceived separation (either frontoparallel or in depth) between the objects (Gogel, 1970). Applying the adjacency principle to induction effects found in geometric illusions, it is expected that either frontoparallel or stereoscopic displacement of the induction and test components will reduce the magnitude of the illusion. Consistent with the adjacency principle, Hennessy and Liebowitz (1972) found that the magnitude of the Ponzo illusion was reduced as the bars (the test objects) were stereoscopically displaced in front of the wedge figure (the induction object). Greene, Lawson, and Godek (1972), although obtaining results consistent with those of Hennessy and Leibowitz for a stereoscopic displacement of the bars in front of the wedge (crossed disparity), found, contrary to the adjacency principle, an increase in the illusion for a stereoscopic displacement of the bars behind the wedge (uncrossed disparity). Green et al. interpret their results in terms of the apparent size of the bars relative to the wedge figure as a function of the stereoscopic displacement of the bars. The present study, paralleling the experiments by Gogel and Koslow (1971, 1972), examines the effect of apparent depth separation

The author wishes to thank Robert E. Newton for his assistance in the collection and analysis of the data. This investigation was supported by United States Public Health Service Research Grant MH 15651 from the National Institute of Mental Health. Requests for reprints should be sent to Walter $C$. Gogel, Department of Psychology, University of California, Santa Barbara, California 93106. between the induction wedge and a pair of test bars both for situations in which only one wedge is present and for situations in which two wedges of opposite orientation are stereoscopically presented at different distances.

A perspective drawing illustrating a situation in which the two wedge figures are presented simultaneously with different stereoscopic positions of a pair of bars is shown in Figure 1. The dashed rectangles enclosing the wedge figures are to maintain the perspective of the drawing for purposes of illustration and were not present during the experiment. The basic Ponzo illusion is that demonstrated by placing two equally sized bars in the plane of the wedge figure. If the wedge figure were absent, it would be expected that the bars would appear to have the same size. But, with the wedge

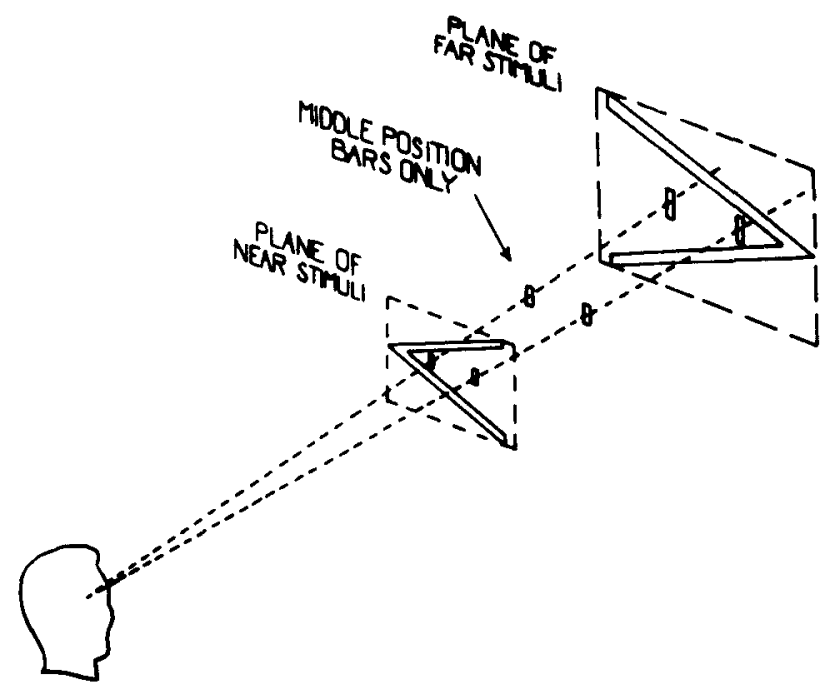

Figure 1. Perspective drawing lllustrating the situation in which two wedges (induction objects) of opposite orientation are presented with three alternative stereoscopic positions of the bars (test objects). 
present. the bar near the apex of the wedge (the apical harl) will appear larger than the other bar (the nonapical bar). Figure 1 illustrates the situation in which a pair of bars is stereoscopically either at the distance of the near wedge, between the two wedges in depth. or at the distance of the far wedge. According to the adjacency principle, the direction of the Ponzo illusion in Figure 1 should be determined mostly by the wedge at the same apparent depth as the pair of bars. In particular. with the wedge orientations shown in Figure 1, the left bar of the pair of bars should appear larger than the right bar when the bars are at the near position and the right bar of the pair should appear larger than the left bar when the bars are at the far position. For the middle position of the pair of bars, the illusion should be intermediate between that obtained at either the near or far positions of the bars.

Figure 2 illustrates the projection of the situation of Figure 1 on a position midway between the eyes of the observer (the cyclopean eye). Since the cyclopean projection can be considered to represent the perceived directional orientation of the stimuli. it is clear that if the perceived depth separation of the bars from the wedge were not an important factor in the Ponzo illusion no illusion would occur in the situation using the oppositely oriented wedges shown in Figure 1.

In addition to using two wedges at different stereoscopic distances with three stereoscopic positions of the pair of bars, two additional situations were used in the present study. In one of these, the wedge figure at the near distance was omitted and in the other the wedge figure at the far distance was omitted. Thus, three basic situations were used with the three alternative stereoscopic positions of the pair of bars: (1) Two wedge figures of opposite orientation were presented at the near and far distances; (2) only one wedge figure was presented with this wedge at the far distance; (3) only one wedge figure was presented with this wedge at the near distance.

The application of the adjacency principle to the situations in which only one wedge was presented requires some additional discussion. The perceived (stereoscopic) displacement of the pair of bars from the distance of the single wedge figure is expected to modify the illusion in the direction expected from the removal of the wedge, i.e., the Ponzo illusion should reduce in magnitude as the stereoscopic separation between the induction and test objects is increased. This would occur, however, only if there were some factor which would make the pair of bars appear to have the same apparent size in the absence of the wedge figure. This factor concerns the effect of perceived distance on perceived size as expressed by the size distance invariance hypothesis (SDIH). According to the SDIH, the bars of equal retinal size

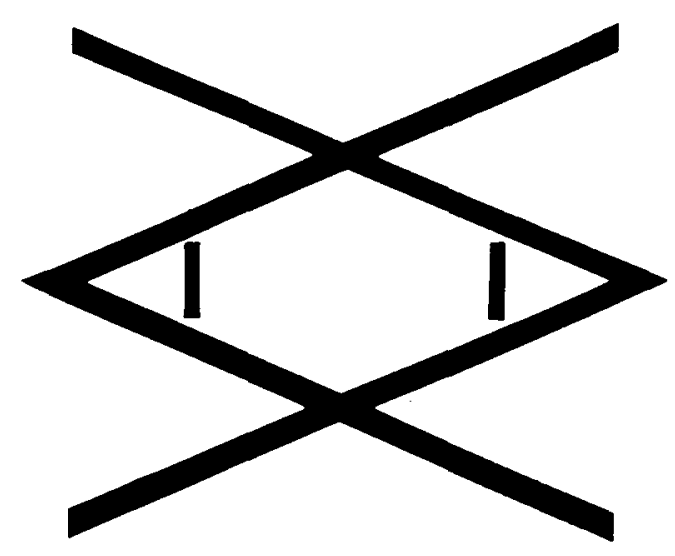

Figure 2. Projection on a cyclopean eye of the situation illustrated in Figure 1.

presented at the same stereoscopic distance (the same perceived distance from the observer) should have the same perceived size. Thus, from the adjacency principle, in the case of the single wedge, all other factors equal, stereoscopic displacement of the pair of bars from the wedge should result in greater effectiveness of the SDIH relative to the effectiveness of the wedge figure in determining the perceived relative sizes of the bars. Basically, a conflict of perceptual information is involved in the situation in which the stereoscopic depth separation between the bars and wedge is varied. In the case of one wedge, the conflict is between the induction effect from the wedge figure and the SDIH. In the case of the two wedges presented simultaneously at opposite orientations (Figure 1), the conflict is primarily between the two opposite induction effects. In the case of either type of conflict, the adjacency principle asserts that the relative weight which the observer gives to a particular source of perceptual information, when more than one source is present simultaneously, varies inversely with the perceived separation of the objects between which this information is occurring. ${ }^{1}$

\section{EXPERIMENT I}

\section{Method}

Apparatus. The wedges and bars were luminous tigures produced by the appropriate masking of luminous surfaces. The visual angular size of the bars always was $11.2^{\prime}$ in width and $1^{\circ} 46.2^{\prime}$ in height. The angular size of the width of the lines (strips) of the wedge tigures always was $21.4^{\prime}$, with the vertical extension of the "nedge $15^{\circ} 8.5^{\circ}$, and with the wedge forming an angle of $41^{\circ} 13.9^{\prime}$. The method of presenting the stimuli is shown in the top view drawing of Figure 3 . The wedge figures were viewed binocularly by means of two partially reflecting, partially transmitting mirrors. The near and far wedge figures were 100 and $160 \mathrm{~cm}$ from the observer, respectively. The wedges were always oriented with apex either left or right. The pair of bars, binocularly viewed through the two mirrors, were physically at a distance of $123 \mathrm{~cm}$ from the 


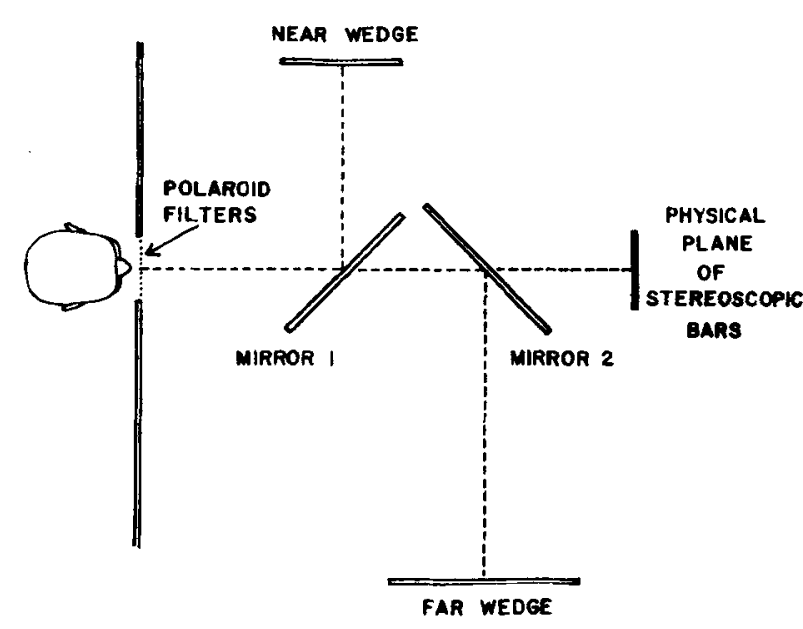

Figure 3. A schematic top view drawing of the apparatus for producing the near and far wedge and the three stereoscopic positions of the bars.

observer (the plane of the stereoscope), were always stereoscopically equidistant, and could be presented as a pair stereoscopically at $100(\mathrm{~N}), 123(\mathrm{M})$, or $160 \mathrm{~cm}(\mathrm{~F})$ from the observer (assuming, consistent with similar groups used previously, an interocular eye distance of $6.2 \mathrm{~cm}$ ). The near and far stereoscopic distances of the pair of bars were produced by means of appropriately oriented Polaroids located at the distance of the plane of the stereoscope and at the observer's eyes. The orientation of the Polaroids at the observer's eyes could be changed by the experimenter from a location outside the observation booth by moving a slide which positioned either one or two pairs of Polaroids in front of the observer's eyes. A top view of the Polaroid stereoscope for presenting the pair of bars at the different distances is shown in Figure 4. Six luminous vertical bars, identical in size and shape (labeled " $a$ " through " $f$ " in Figure 4), were produced in the plane of the stereoscope. For the middle distance $(123 \mathrm{~cm})$, only the bars labeled " $b$ " and " $e$ " were visible and were viewed by both eyes without the Polaroids. As indicated by the filled dot surrounded by the circle, these bars were physically and stereoscopically at the plane of the stereoscope. To produce the near distance, only a and $d$ were seen with the right eye and only $c$ and $f$ with the left eye. For the far distance of the bars, only $c$ and $f$ were seen with the right eye and only $a$ and $d$ with the left eye. The brightness of the various bars and the wedges was adjusted by the experimenter to a value of $.3 \mathrm{fL}$ as viewed from the observation position. Since the accommodative distance of the bars was always to $123 \mathrm{~cm}$, the accommodative difference in diopters between a pair of bars and either wedge was always the same (about .2 D), although opposite in direction for the near and far wedge. The stereoscopic difference between the near and far stimulus positions (of wedge or bars) was $80^{\prime}$ of binocular disparity, with the middle position $40^{\prime}$ behind and in front of the near and far position, respectively. The two wedges could be turned on or off independently, with the following six possible experimental situations presented with each of the three stereoscopic positions of the bars: Two wedges presented simultaneously with (1) near wedge pointing left (apex left), far wedge pointing right (apex right) or (2) near wedge pointing right, far wedge pointing left. One wedge at the far position only and pointing (3) left or (4) right. One wedge at the near position only and pointing (5) left or (6) right. Control conditions consisted of presenting the three stereoscopic positions of the pair of bars in the absence of any wedge figures.

The stimuli were viewed from a booth that was totally dark during the observations. A lighted surface of $610 \mathrm{fL}$ was located on the side wall in order to light-adapt the observer before beginning the experiment and between presentations of the experimental and control conditions. The stimuli were viewed through an aperture which was occluded by the experimenter between presentations by closing was occluded by the experimenter between presentations by luminous wedges and bars. During the experiment, the experimenter and the observer communicated by means of a headphone and microphone. White noise was presented in the headphones between trials to mask any sounds made by the experimenter. In addition to the viewing aperture, the observation position contained an adjustable stool, the slide for the Polaroids, and a head- and chinrest. The observer received general instructions regarding his task before entering the observation booth and specific instructions thereafter.

Observations. The observers were 56 men and 88 women, who partially satisfied a requirement of an undergraduate course in psychology by participating in the experiment. All had an acuity of $20 / 30$ both near and far and a stereoacuity of $18 \mathrm{sec}$, as measured with a Keystone orthoscope. The average internodal distance of the observers, as measured with a Mark I interpupillometer, was $6.19 \mathrm{~cm}$ with a range of 5.4 to $6.9 \mathrm{~cm}$.

Procedure. In the experimental conditions, either one or two wedges were presented in addition to the pair of bars presented successively at the three stereoscopic distances. Half of the observers (72) were presented with the two wedges, and the remaining 72 observers with one wedge only. For 36 of the observers presented with the two wedges simultaneously, the near wedge pointed to the left and the far wedge pointed to the right. For the other 36 observers, the near wedge pointed right and the far wedge pointed left. The 72 observers presented with only one wedge were divided into four groups of 18 observers. With one group, the near wedge pointed to the left; with another group, the near wedge pointed to the right; with another group, the far wedge pointed to the left; and with another group, the far wedge pointed to the right. A trial in the experimental condition consisted of verbal reports of the apparent distance $\left(D^{\prime}\right)$ of the wedge and of the apparent distances $\left(D^{\prime}\right)$ and apparent relative sizes $\left(S^{\prime}\right)$ of the bars. Thus, each observer in each experimental condition was presented with three trials. The order of presenting the three stereoscopic positions of the bars (one position for each trial) was systematically varied between observers. The distance judgments of either a bar or a wedge were

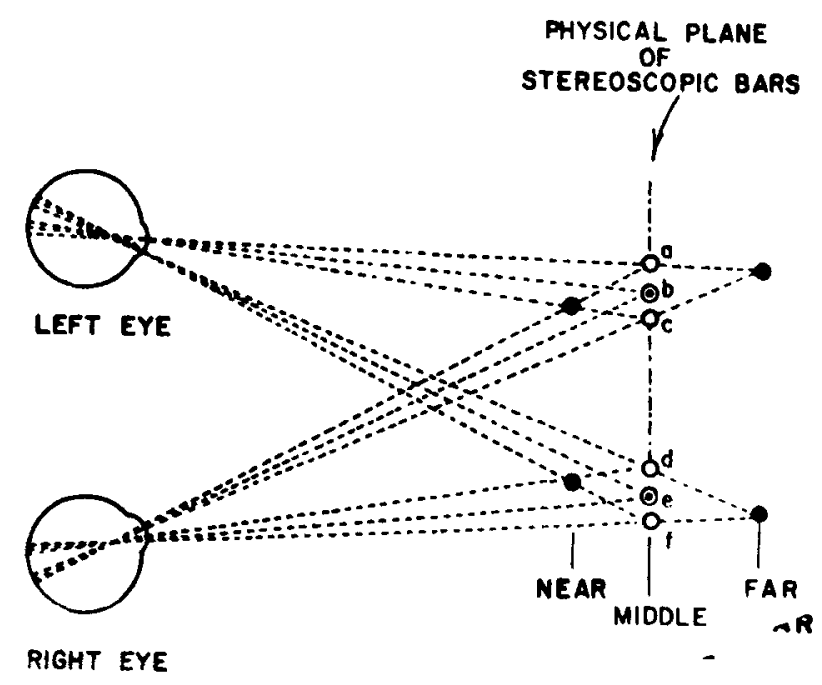

Figure 4. A schematic top view drawing of the Polaroid stereoseope used in producing the three stereoscopic positions of the pair of bars. 
whained by asking the observer to indicate verbally in feet or inches. or in some combination of both, the perceived distances of each of these objects from his eves. For the size judgments. one of the two hars (the standard) on each trial was assigned arbitrarily a value of 10 and the observer was asked to assign a value to the other bar (using fractions if necessary) which indicated the perceived height of this bar relative to the standard. For example, a report of 7.5 would indicate that the nonstandard bar in a pair of bars was perceived to be three-quarters as tall as the standard bar. The standard bar was either the right or the left bar of the pair of bars for a particular observer throughout the experiment, but was varied between observers, with the result that for each condition the standard was the right or the left bar (or the apical or nonapical harl equally often. The order in which the observer reported the perceived distance of the wedge or wedges, the perceived distances of each of the bars of a pair, and the perceived ratio of the sizes of the bars was systematically varied between observers.

The control conditions, which always followed the experimental conditions. consisted of reports of the perceived distances and perceived size ratios of the bars for the three distance positions of the bars in the absence of any wedges. The purpose of the control conditions was to determine whether there was any systematic tendency to perceive one bar of a pair as being at a different distance or as having a different size than the other in the absence of any induction object for any of the three distance positions of the bars.

\section{Results}

The results from the reports of relative size are summarized in Table 1 . The $S^{\prime}$ ratio is the reported size of one bar relative to the other bar. To compare $S^{\prime}$ ratios between different situations, a procedure for calculating these ratios was used which can be discussed with the aid of Figure 1. In the situation illustrated by Figure 1 , the computed $S^{\prime}$ ratio, for each of the three distance positions of the bars, is the perceived size of the right bar divided by the perceived size of the left bar. For example, if a response had been that the left bar was three-quarters the size of the right bar (the right bar is the standard in this example), the computed $S^{\prime}$ ratio would be 1.33 . This procedure was used with (1) the simultaneous presentation of the two wedges for the case in which the near wedge pointed left and the far wedge pointed right, (2) the presentation of only the near wedge pointing left, and (3) the presentation of only the far wedge pointing right. The procedure for calculating the $S^{\prime}$ ratios for the remaining situations for each of the three distances of the bars was to divide the perceived size of the left bar by the perceived size of the right bar. The three situations to which this procedure was applied were (4) the simultaneous presentation of the two wedges for the case in which the near wedge pointed right and the far wedge pointed left, (5) the presentation of only the near wedge pointing right, and (6) the presentation of only the far wedge pointing left. This procedure permitted the $S^{\prime}$ ratios obtained for a particular distance of the bars (N. M, or F) to be averaged for a particular situation (one wedge only, near; one wedge only, far; or two wedges, one near and one far), regardless of the direction in which the wedge or wedges pointed and regardless of whether the standard was the right or left bar.

Table 1 also gives the order of $S^{\prime}$ ratios expected from the Ponzo illusion and from the depth displacement of the bars with respect to the wedges. Consider the case of one wedge only at the near distance. From the procedure for calculating $S^{\prime}$ ratios described above, regardless of the direction in which the near wedge pointed, the $S^{\prime}$ ratio was the perceived size of the nonapical bar divided by the perceived size of the apical bar. Thus, all $S^{\prime}$ ratios should have been less than unity according to the Ponzo illusion and should increase (toward unity) as the bars were increasingly displaced behind the wedge. Consider the case of one wedge only at the far distance. In this case, the $S^{\prime}$ ratio resulted from dividing the perceived size of the apical bar by that of the nonapical bar (regardless of the direction in which the far wedge pointed). Thus, all $S^{\prime}$ ratios should be greater than unity according to the Ponzo illusion and should decrease (toward unity) as the bars were increasingly displaced in front of the wedge. Consider the case in which the two wedges were presented simultaneously. In this case, only if the stereoscopic displacement of the bars from a wedge were a significant factor would an illusion of

Table 1

Results from Experimental Conditions of Experiment 1: Reported Ratios of Sizes $\left(S^{\prime}\right)$ of Bars as a Function of Their Stereoscopic Position at a Near (N), Middle (M), or Far (F) Distance, With Either One Wedge Presented (at $N$ or $F$ ) or Two Wedges Presented Simultaneously (One at $N$ and the Other at $F$ )

\begin{tabular}{|c|c|c|c|c|c|c|c|c|c|}
\hline \multirow[b]{3}{*}{ Distance of Bars } & \multicolumn{6}{|c|}{ One Wedge Only } & \multirow{2}{*}{\multicolumn{3}{|c|}{$\begin{array}{l}\text { Two Wedges Presented } \\
\text { Simultaneously } \\
\text { (One Near, One Far) }\end{array}$}} \\
\hline & \multicolumn{3}{|c|}{ Wedge Near } & \multicolumn{3}{|c|}{ Wedge Far } & & & \\
\hline & $\mathrm{N}$ & $\mathbf{M}$ & $\mathrm{F}$ & $\mathrm{N}$ & M & $\mathbf{F}$ & $\mathbf{N}$ & $\mathbf{M}$ & $\mathrm{F}$ \\
\hline Mean $S^{\prime}$ Ratios & .94 & .94 & .93 & 1.04 & 1.03 & 1.16 & .90 & .94 & 1.08 \\
\hline Median S' Ratios & .93 & .95 & .96 & 1.00 & 1.00 & 1.19 & .91 & .95 & 1.05 \\
\hline$\sigma$ of $S^{\prime}$ Ratios & .12 & .12 & .09 & .11 & .08 & .10 & .12 & .09 & .11 \\
\hline Expected $S^{\prime}$ Ratios & \multicolumn{3}{|c|}{$\mathrm{N}<\mathrm{M}<\mathrm{F}<1.0$} & \multicolumn{3}{|c|}{$1.0<\mathrm{N}<\mathrm{M}<\mathrm{F}$} & \multicolumn{3}{|c|}{$1.0>\mathrm{N}<\mathrm{M}<\mathrm{F}>1.0$} \\
\hline Mean Rank of $S^{\prime}$ Ratios & 2.01 & 1.92 & 2.07 & 1.62 & 1.69 & 2.69 & 1.50 & 1.76 & 2.74 \\
\hline Expected Ranks & \multicolumn{3}{|c|}{$2.0>\mathrm{N}<\mathrm{M}<\mathrm{F}>2.0$} & \multirow{2}{*}{\multicolumn{3}{|c|}{$\begin{array}{l}2.0>\mathrm{N}<\mathrm{M}<\mathrm{F}>2.0 \\
x_{r}^{2}=26.2, \mathrm{p}<.001\end{array}$}} & \multirow{2}{*}{\multicolumn{3}{|c|}{$\begin{array}{l}2.0>\mathrm{N}<\mathrm{M}<\mathrm{F}>2.0 \\
\chi_{\mathrm{r}}^{2}=58.0, \mathrm{p}<.001\end{array}$}} \\
\hline Friedman Analysis of Ranks & $x_{r}^{2}=$. & & & & & & & & \\
\hline
\end{tabular}


Table 2

Results from Experimental Conditions of Experiment 1: Reported Distances $\left(D^{\prime}\right)$ in Centimeters of Bars and Wedges for the Three Stereoscopic Positions of the Bars, Near (N), Middle (M), and Far (F), and the Two Stereoscopic Positions of the Wedges, With Either One Wedge Presented Alone (at $\mathbf{N}$ or $\mathbf{F}$ ) or With Both Wedges Presented Simultaneously (at N and F)

\begin{tabular}{|c|c|c|c|c|c|c|c|c|c|}
\hline \multirow[b]{2}{*}{ Distance of Bars } & \multicolumn{3}{|c|}{ Mean $D^{\prime}$} & \multicolumn{3}{|c|}{ Median $D^{\prime}$} & \multicolumn{3}{|c|}{$\sigma$ of $\mathrm{D}^{\prime}$} \\
\hline & $\mathrm{N}$ & $\mathbf{M}$ & F & $\mathrm{N}$ & $\mathbf{M}$ & $\mathbf{F}$ & $\mathbf{N}$ & $\mathbf{M}$ & $\mathrm{F}$ \\
\hline \multicolumn{10}{|l|}{ Near Wedge Only* } \\
\hline $\begin{array}{l}\text { Apical Bar } \\
\text { Other Bar } \\
\text { Near Wedge }\end{array}$ & $\begin{array}{r}170 \\
180 \\
93\end{array}$ & $\begin{array}{r}152 \\
158 \\
78\end{array}$ & $\begin{array}{r}232 \\
236 \\
73\end{array}$ & $\begin{array}{l}86 \\
91 \\
91\end{array}$ & $\begin{array}{r}91 \\
109 \\
76\end{array}$ & $\begin{array}{r}122 \\
136 \\
61\end{array}$ & $\begin{array}{r}489 \\
489 \\
59\end{array}$ & $\begin{array}{r}240 \\
239 \\
41\end{array}$ & $\begin{array}{r}483 \\
483 \\
30\end{array}$ \\
\hline \multicolumn{10}{|l|}{ Far Wedge Only } \\
\hline $\begin{array}{l}\text { Apical Bar } \\
\text { Other Bar } \\
\text { Far Wedge }\end{array}$ & $\begin{array}{r}69 \\
71 \\
165\end{array}$ & $\begin{array}{l}102 \\
103 \\
164\end{array}$ & $\begin{array}{l}121 \\
127 \\
114\end{array}$ & $\begin{array}{r}65 \\
74 \\
152\end{array}$ & $\begin{array}{r}91 \\
91 \\
152\end{array}$ & $\begin{array}{r}107 \\
118 \\
93\end{array}$ & $\begin{array}{l}26 \\
26 \\
66\end{array}$ & $\begin{array}{l}47 \\
47 \\
70\end{array}$ & $\begin{array}{l}61 \\
65 \\
56\end{array}$ \\
\hline \multicolumn{10}{|l|}{$\begin{array}{l}\text { Two Wedges Presented } \\
\text { Simultaneously }\end{array}$} \\
\hline $\begin{array}{l}\text { Left Bar } \\
\text { Right Bar } \\
\text { Near Wedge } \\
\text { Far Wedge }\end{array}$ & $\begin{array}{r}77 \\
74 \\
76 \\
159\end{array}$ & $\begin{array}{r}112 \\
114 \\
82 \\
167\end{array}$ & $\begin{array}{r}164 \\
160 \\
71 \\
151\end{array}$ & $\begin{array}{r}62 \\
61 \\
61 \\
137\end{array}$ & $\begin{array}{r}91 \\
91 \\
61 \\
152\end{array}$ & $\begin{array}{r}124 \\
130 \\
61 \\
122\end{array}$ & $\begin{array}{l}40 \\
37 \\
45 \\
93\end{array}$ & $\begin{array}{r}93 \\
99 \\
72 \\
166\end{array}$ & $\begin{array}{r}163 \\
153 \\
36 \\
135\end{array}$ \\
\hline
\end{tabular}

* Discrepancies between means and medians and the large $\sigma$ s for the bars in this situation are due to the data from one observer.

size occur for any stereoscopic position of the bars. Again, from the above method of calculating S' ratios, if the $S^{\prime}$ ratio was determined by the wedge at the same plane as the bars, it should increase from the near to the far position and it should be less than unity at the near position, greater than unity at the far position, and between these two values at the middle position.

A rank order analysis was applied to the $S^{\prime}$ ratios as a function of the three stereoscopic distances of the pairs of bars. The largest of the three ratios from a particular observer (for a particular situation) was given the rank of 3 , with the smallest given the rank of 1. The magnitude of the mean rank as a function of the stereoscopic position of the bar is shown in Table 1 for each of the three basic situations of the experiment.

As indicated by Table 1, in agreement with the results expected from the Ponzo illusion, the apical bar appeared larger than the nonapical bar of the same physical size and distance in the case in which either the near wedge or the far wedge was presented alone. Also, as indicated by the Friedman two-way analysis of the rank data, the stereoscopic displacement of the bars from the plane of the single wedge decreased the magnitude of the illusion for the case in which the displacement was in front of the far wedge presented alone but not when the displacement was behind the near wedge presented alone. The use of the far, but not the near, wedge indicates a reduction in the magnitude of the induction effect as the test object is displaced from the plane of the induction object. The importance of depth separation is strongly supported by the $\mathrm{S}^{\prime}$ ratios obtained from the simultaneous presentation of the two wedges. In this case, a ratio less than unity at the near distance of the bars indicates that the near (not the far) wedge determined the direction of the illusion when the bars were stereoscopically (and perceptually) at the plane of the near wedge. A ratio greater than unity at the far distance of the bars indicates that the far wedge determined the direction of the illusion when the bars were stereoscopically at the plane of the far wedge. If the depth separation of the bars from a particular wedge significantly reduced the induction effect of that wedge, both the $S^{\prime}$ ratios and the rank order of the $S^{\prime}$ ratios should have increased from $N$ to $F$. Clearly this occurred.

The reported distances of the bars and wedge figures are summarized in Table 2 . As expected, the changes in the stereoscopic positions of the bars resulted in a change in their perceived distance relative to the wedges. It should be noted, however, that in the conditions in which only one wedge was presented the apparent distance of the bars for a particular stereoscopic position tended to be less when the wedge was at the far, rather than at the near, distance. Similarly, the apparent distance of a single wedge tended to decrease with increases in the stereoscopic distance of the pair of bars. It seems that the change in the stereoscopic distance of the bars for a constant distance of a wedge resulted in a change in opposite directions of the perceived distance from the observer of both the bars and the wedge. As expected, this effect did not occur when the two wedges were presented simultaneously. These results are consistent with the finding (Gogel, 1972b) that when only stereoscopic cues of distance are available there is a 
Table 3

Results from Control Conditions in Experiment 1 (No Wedges

Present): Reported Distances $\left(D^{\prime}\right)$ in Centimeters and Ratios of Reported Sizes ( $\left.S^{\prime}\right)$ of Bars

\begin{tabular}{|c|c|c|c|c|c|c|c|c|c|}
\hline \multirow[b]{3}{*}{ Bars } & \multicolumn{6}{|c|}{$D^{\prime}$ of Bars } & \multirow{2}{*}{\multicolumn{3}{|c|}{$\begin{array}{l}S^{\prime} \text { Ratio of Bars } \\
\text { Right Bar/Left Bar }\end{array}$}} \\
\hline & \multicolumn{3}{|c|}{ Left Bar } & \multicolumn{3}{|c|}{ Right Bar } & & & \\
\hline & Mean & $M d n$ & $\sigma$ & Mean & Mdn & $\sigma$ & Mean & Mdn & $\sigma$ \\
\hline $\mathbf{N}$ & 119 & 91 & 137 & 11 & 91 & 136 & 1.01 & 1.00 & .11 \\
\hline M & 131 & 116 & 105 & 130 & 112 & 102 & 1.00 & 1.00 & .10 \\
\hline $\mathrm{F}:$ & 142 & 122 & 91 & 139 & 122 & 91 & .99 & 1.00 & .10 \\
\hline
\end{tabular}

position within the binocular configuration which tends to remain invariant in perceived distance with changes in the stereoscopic depth within the configuration, regardless of whether the near or far part of the configuration is stereoscopically moved. In studies of size and distance in binocular configurations, sometimes a portion of the stimulus configuration is used as a standard for judging the sizes and distances of other portions of the contiguration as these other portions are moved to different stereoscopic locations (see Greene et al.. 1972). The above results indicate that this procedure should be avoided since it is unlikely that such a standard would remain unchanged in either perceived distance or perceived size as a function of the stereoscopic changes in the other portions of the display.

The reported distances and relative sizes of the bars in the absence of wedges (the control conditions) are summarized in Table 3 . It is evident that under these conditions the perceived sizes of the left and right bar were equal at each of the three stereoscopic distances. Since no wedge was present, binocular disparity was absent between the bars and any other object, with convergence the only cue to the distance of the bars. The $\mathrm{D}^{\prime}$ data of Table 3 indicate that decreases in convergence were somewhat effective in producing increases in perceived distance. Also, as expected, the ratio of the perceived sizes of the bars was always approximately unity.

\section{EXPERIMENT II}

The results from the conditions in which the two wedges were presented simultaneously provide strong evidence that depth adjacency between the test and induction objects is an important factor in determining the magnitude and direction of the Ponzo illusion. Depth adjacency can also be used to explain the clear decrease in the illusion when the bars were displaced stereoscopically in front of the induction figure and the only wedge present was at the far distance. But the lack of decrease in the magnitude of the illusion when the bars were displaced behind the wedge under the condition in which the single wedge was at the near position is consistent with neither the fictor of depth adjacency nor with the results of Greene et al. (1972). It will be noted, however, that, for the condition in which only a single wedge was present, the magnitude of the illusion when the bars "ice stereoscopically equidistant with this wedge was $6{ }^{\prime \prime}$ or $100(1-.94)$ when the wedge was at the near position and $16 \%$ or $100(1.16-1.00)$ when the wedge was at the far position. Thus, in the former case the magnitude of the Ponzo illusion was small even under the optimal condition of stereoscopic equidistance between the bars and wedge. In Experiment II, the situation in which the bars were stereoscopically at the distance of the single wedge or $40^{\prime}$ or $80^{\prime}$ behind this wedge again was investigated, but in this case the nedge was at 160 rather than $100 \mathrm{~cm}$ from the observer. For this purpose, the plane of the Polaroid stereoscope was moved to $229 \mathrm{~cm}$ from the observer. and the bars on this plane were moditied in separation and size so as to produce pairs of bars of the same retinal sise as in Experiment 1 , but at stereoscopic distances of 160,229 , or $400 \mathrm{~cm}$ from the observer. The orientation of the wedge was to the right with half the observers and to the left with the remaining observers. Following the presentation of the experimental conditions, all observers were presented with the control in which the wedge was absent. The procedure and instructions in Experiment II were identical to those used for the single near wedge condition of Experiment I.

The results from the experimental and control conditions of Experiment II are shown in Table 4. The only difference between the meaning of the notation used in Table 4 and in the previous tables is that, in Table 4. N. M, and F reter to 160,229 , and $400 \mathrm{~cm}$ from the observer rather than to the 100, 123 . and $160 \mathrm{~cm}$ distances used in Experiment $I$.

The $S^{\prime}$ ratios in Table 4 are similar to those for the near single wedge in Table 1 in that no systematic change in the magnitude of the Ponzo illusion occurred with uncrossed disparity of the bars. The $D^{\prime}$ data of Table 4 confirm that the perceived depth between the wedge and pair of bars increased with increasing anounts of uncrossed disparity and that the wedge, despite its constant distance, tended to appear closer as the stereoscopic distance of the pair of bars increased.

\section{DISCUSSION}

Clear support for the adjacency principle as applied to the Ponzo illusion occurred in the situation in Experiment $I$ in which two wedges of opposite orientation were presented at different distances. An explanation in terms of the changes in the perceived sizes of the bars relative to the wedges with changes in the perceived distances of the bars as suggested by 
Table 4

Results from Experiment 2 from Both the Experimental and Control Conditions ( ${ }^{\prime}$ in Centimeters)

\begin{tabular}{|c|c|c|c|c|c|c|c|c|c|c|c|c|}
\hline \multirow[b]{2}{*}{ Distance of Bars } & \multicolumn{3}{|c|}{$\begin{array}{l}\mathrm{S}^{\prime} \text { Other Bar/ } \\
\mathrm{S}^{\prime} \text { Apical Bar }\end{array}$} & \multicolumn{3}{|c|}{ D' Apical Bar } & \multicolumn{3}{|c|}{$D^{\prime}$ Other Bar } & \multicolumn{3}{|c|}{$\mathrm{D}^{\prime}$ of Wedge } \\
\hline & $\mathrm{N}$ & M & $\mathrm{F}$ & $\mathrm{N}$ & $\mathrm{M}$ & $\mathbf{F}$ & $\mathrm{N}$ & $\mathbf{M}$ & $\mathrm{F}$ & $\mathrm{N}$ & $\mathbf{M}$ & $\mathrm{F}$ \\
\hline \multicolumn{13}{|l|}{ Wedge Present } \\
\hline $\begin{array}{l}\text { Mean } \\
\text { Median } \\
\sigma \\
\text { Rank } \\
\text { Friedman Analy sis }\end{array}$ & $\begin{array}{l}.91 \\
.91 \\
.11 \\
2.03 \\
x_{r}^{2}=5\end{array}$ & $\begin{array}{r}.89 \\
.90 \\
.12 \\
1.81 \\
.34, \mathrm{p}\end{array}$ & $\begin{array}{r}.93 \\
.93 \\
.13 \\
2.16 \\
.05\end{array}$ & $\begin{array}{r}115 \\
107 \\
55\end{array}$ & $\begin{array}{l}177 \\
152 \\
101\end{array}$ & $\begin{array}{l}217 \\
183 \\
130\end{array}$ & $\begin{array}{r}120 \\
110 \\
57\end{array}$ & $\begin{array}{l}188 \\
152 \\
104\end{array}$ & $\begin{array}{l}229 \\
183 \\
144\end{array}$ & $\begin{array}{r}121 \\
122 \\
60\end{array}$ & $\begin{array}{l}98 \\
91 \\
46\end{array}$ & $\begin{array}{l}83 \\
91 \\
33\end{array}$ \\
\hline & \multicolumn{3}{|c|}{$\begin{array}{l}S^{0} \text { Right Bar } \\
S^{\prime} \text { Left Bar }\end{array}$} & & Left $B$ & & \multicolumn{3}{|c|}{ D' Right Bar } & & & \\
\hline \multicolumn{13}{|l|}{ Wedge Absent } \\
\hline $\begin{array}{l}\text { Mean } \\
\text { Median } \\
\sigma\end{array}$ & $\begin{array}{r}1.01 \\
1.00 \\
.10\end{array}$ & $\begin{array}{r}1.00 \\
1.00 \\
.08\end{array}$ & $\begin{array}{r}1.04 \\
1.00 \\
.14\end{array}$ & $\begin{array}{l}165 \\
122 \\
124\end{array}$ & $\begin{array}{r}177 \\
152 \\
90\end{array}$ & $\begin{array}{l}199 \\
183 \\
110\end{array}$ & $\begin{array}{l}160 \\
122 \\
113\end{array}$ & $\begin{array}{r}173 \\
147 \\
87\end{array}$ & $\begin{array}{l}194 \\
183 \\
104\end{array}$ & & & \\
\hline
\end{tabular}

Greene et al. (1972) seems unable to account for the reversal in the direction of the Ponzo illusion that occurred under this condition.

When a single wedge was used, the decrease in the magnitude of the illusion as a function of increasing apparent displacement of the bars in front of the wedge is expected from the adjacency principle and, according to Greene et al. (1972), this also is expected from the change in the perceived size of bars resulting from the change in perceived distance. For the case in which the bars were stereoscopically displaced behind the single wedge (in either Experiment I or Experiment II) neither the increase in the illusion expected by Greene et al. nor the decrease in the illusion expected from the adjacency effect occurred. There are several alternative explanations of this latter result. It is possible that the effects of depth adjacency and apparent size, since they were in opposite directions, cancelled each other. It also is possible that an attention effect occurred such that the wedge could be less readily ignored when the bars were perceptually behind rather than in front of the wedge. A third possibility, which is favored by this author, concerns the description of the adjacency principle in terms of cue conflicts. In the case of the two wedges of opposite orientations presented simultaneously, the illusion involved mainly a conflict between the induction effects from each of the two wedges. In the case in which only a single wedge was present, the cue conflict was between the perceived equality in the relative sizes of the bars expected from the SDIH and the perceived inequality expected from the wedge. Possibly the zero binocular disparity between the bars of the pair (which normally would have produced a perceived equality in $S^{\prime}$ ) was more effective for near distances of the bars than for far distances. This possibility is supported by the greater magnitude of the Ponzo illusion that occurred in
Table 1 when both the bars and single wedge were at a far, as compared with a near, distance. From this result, it is reasonable to expect that displacing the pair of bars in front of the single wedge would produce more effective information of size equality to counteract the Ponzo illusion than would displacement behind the wedge figure. Or, stated more specifically, the increasing perceived separation between the bars with increasing perceived distance of the bars would, according to the adjacency principle, result in a decreased effectiveness of the zero disparity between the pair of bars in making the bars appear equal in size. Thus, displacement of the pair of bars behind the wedge simultaneously would decrease both the effectiveness of the induction from the wedge and the effectiveness of perceived distance on perceived size. Conversely, it is also possible that stereoscopically positioning the bars in front of the wedge simultaneously increased the contribution of the SDIH and decreased the induction effect, with the result that the magnitude of the Ponzo illusion rapidly decreased with crossed disparity.

The results from the present study are consistent with the studies on induced motion as a function of depth separation by Gogel and Koslow $(1971,1972)$. Like the present results, changes in the direction of the illusion were found when opposed induction effects were present at different distances. Also, a clear decrease in the magnitude of the induced motion for stereoscopic displacement of the test object in front of the induction object was found, whereas displacement behind the induction object resulted in a smaller or no decrease in the illusion. In the study by Gogel and Koslow (1971), this latter result was interpreted as being in agreement with the factor of perceived depth in that, from the SDIH, an increase in the magnitude of the induction effect would be expected with increasing perceived distances of the 
test object. Reductions in the magnitude of the induction effects dependent only on the magnitude, and not the direction, of the depth separation of the induction and test object have been found, however, "ith simultaneous color contrast (Mershon. 1972; Wist d Susen. 1973) and the binocular induction of depth associated with the trapezoidal window (Gogel, 1972a). From these and the present study, it seems clear that. in agreement with the adjacency principle, induction effects can be modified by the depth separation of the test and induction object.

\section{REFERENCES}

Gogel, W. C. Adjacency principle and three-dimensional visual illusion. In J. C. Baird (Ed.), Human space perception Proceedings of the Dartmouth Conference. Psychonomic Monograph Supplements, 3(13, Whole No. 45), 1970.

GOGEL, W. C. Depth adjacency and cue effectiveness. Journal of Experimental Psychology, 1972, 92, 176-181. (a)

Gogel, W. C. Scalar perceptions with binocular cues of distance. American Journal of Psychology, 1972, 85, 477-498. (b)
Gogel, W. C. Relative motion and the adjacency principle. Quarterly Journal of Experimental Psychology, 1974, 26, 425-437.

Gogel, W. C., \& Koslow, M. The effect of perceived distance on induced movement. Perception \& Psychophysics, $1971,10,142-146$.

Gogel, W. C., \& Koslow, M. The adjacency principle and induced movement. Perception \& Psychophysics, 1972, 11, 309-314.

Greene, R. T., Lawson, R. B., \& Godek, C. L. The Ponzo illusion in stereoscopic space. Journal of Experimental Psychology, 1972, 95, 358-364.

Hennessy, R. T., \& Leibowitz, H. W. Perceived vs, retinal relationships in the Ponzo illusion. Psychonomic Science, 1972. 28. $111 \cdot 112$.

Mershon, D. H. Relative contributions of depth and directional adjacency to simultaneous whiteness contrast. Vision Research, 1972. 12, 969-979.

WIST, E. R., \& SusEn, P. Evidence for the role of post-retinal processes in simultaneous contrast. Psychologische Forschung, $1973,36,1-12$

\section{NOTE}

1. Adjacency effects expressed as a resolution of cue conflicts and applied to the perception of motion are discussed in a study by Gogel (1974).

(Received for publication March 1, 1974; revision received July 26,1974 .) 